mental design, regression and correlation, growth functions and econometrics. The examples are numerous and virtually all taken from forestry problems. The text is well illustrated by diagrams and figures. Inevitably, in intending the book for "students, practical workers and research project leaders", the author will not completely satisfy each category. It is probably too complex for the practical worker and yet not detailed enough for someone engaged in research. To many forest biometricians there will not be enough emphasis on modern computing methods; there is only a brief mention of electronic computers and much of the book, particularly the chapters on regression and correlation, would benefit from a greater bias towards their use. The space devoted to the various subjects is perhaps out of balance, and not related to their importance and degree of use. For example, the analysis of varianee, a technique used very widely in forest research, does not get a wide coverage and contains very little on testing procedures, whereas there is a large amount on distributions which could probably be reduced.

The figures and tables in the translation have been excellently reproduced and anglicized, several of the tables being re-cast to avoid dividing them. Some of the original typographical errors have been corrected but others still exist. The translation of the German text is good with very few ambiguities, such as where, for example, the coefficient of determination is referred to as the coefficient of dependence. The text and formulae are clear and easily followed. This book is a very valuable addition to the bookcase of the research worker and the examples also provide valuable teaching material. H. L. WRIGHT

\section{CRYSTALS, LIQUIDS AND GLASSES}

Physical Properties of Molecular Crystals, Liquids, and Glasses

By A. Bondi. (Wiley Series on the Science and Technology of Materials.) Pp. xix +502 . (Wiley: New York and London, 1968.) 175s.

THIs work attempts to present generalized correlations that should enable chemists and chemical engineers to relate certain physical properties of condensed phases to molecular structure. The physics of molecular condensed phases is not sufficiently well developed theoretically to allow the calculation of precise physical properties from molecular structure data alone. The author has therefore selected the procedure of producing easily usable methods that yield information rapidly and of an order of precision warranted by users who want to estimate physical properties for development and process work. The method adopted was one of extrapolation from known data based on the principles of similarity analysis. Available theory is used first to obtain accessible measures of the important properties of individual molecules and these parameters are then used under guidance of theory to make the physical properties and state variables of interest dimensionless. The resulting plot of experimental data, an application of similarity to molecular physics, forms the basis of the correlations presented in this book. Most of the correlations are based on the use of experimentally accessible properties as correlating parameters, the van der Waals dimensions and volumes, standard entropy of vaporization of liquids and standard heat of sublimation of solids. The group increments for these and related propertios are summarized in table form in chapter 14 .

The states of matter treated are molecular crystals, liquids and glasses. The physical properties considered are the pressure, volume and temperature and related thermal properties and transport properties which yield to the method of treatment. After opening chapters on the properties of molecules and the principle of corresponding state the following chapters deal with the thermal, elastic and transport properties of molecular crystals including polymer crystals. Other chapters cover the subjects of fusion, liquids, p.v.T properties of liquids, heat capacities of liquids and polymer melts, thermal conductivity of non-associated liquids, diffusion, viscosity and finally the physical properties of molecular glasses. The last section of the book collects together calculating methods for physical properties in a form convenient for translation into computer programs.

The book contains an immense amount of valuable information and the author has succeeded in his primary purpose of presenting calculating schemes which will be most valuable to the chemist and chemical engineer in estimating property data required for synthesis and development of new materials and for process calculations and product development work. An additional feature of the book is that in order to assist the above types of users most of the correlations described in the text are restated as estimating methods at the end of each major chapter.

C. E. H. BAwN

\section{PHYSICS HANDBOOK}

\section{Physics Pocketbook}

Edited by Hermann Ebert. Pp. 575. (Oliver and Boyd: Edinburgh and London, 1967.) $63 s$.

EBERT's Physikalische Taschenbuch is somewhat of a German classic. It was first published in 1951 and sub. sequent editions appeared in 1957 and 1962 . The present volume would appear to be a remarkably good translation of the 1962 edition for the references do not go beyond 1960. The translator is not identified nor is there an introduction or preface. The author, or rather compiler, is Hermann Ebert, a member of the Physikalisch-Tech. nische Bundesanstalt, and he therefore speaks with authority in the sections on vacuum technology, hygrometry, thermal expansion, and equations of state of solids which he himself has written. A variety of authors have contributed the numerous other sections and though it would seem they have been given free choice it is remark. able how well a uniform standard is attained throughout the volume.

Naturally, with a limit of six hundred pages and thirteen chapters much has had to be omitted or compressed, but a large amount of accurate information, numerous formulae, and many tables of data have been included. There are deficiencies-some gross, some minor. Not all the information is up to date; for example, the maser is briefly dealt with, but not the laser (neither "molecular amplifier" nor "maser" occur in the index). The recommendations of recent meetings of the Conference of Weights and Measures do not appear. References are nearly all confined to a name and year; for example (Casimir, 1945). Many names are incorrectly spelt, either in the text or in the index.

The subject arrangement of the text is useful, but the numbering of the chapters, sections and subsections is somewhat strange. The first four chapters dealing with units, mathematical aids and statistics are numbered 111 to 114 , and the remaining nine chapters dealing respectively with relativity and quantum theory, mechanics, acoustics, optics, heat, electricity, magnetism and electromagnetism, atomic and nuclear physics, and strength of materials, 120 to 128 . Decimal numbers denote the sections and subsections of the chapters.

The publisher recommends the book as indispensable to all university students. Though students and research workers will find the work informative and stimulating, they should be recommended to use it with care and only in conjunction with other handbooks and encyclopaedias. The trouble is that too much has been attempted-a dictionary, handbook and encyclopaedia cannot all be provided within one volume of six hundred pages. 